\title{
Tumour markers in gastrointestinal malignancy: What use to the clinician?
}

\author{
Bruce R YaCyshyn, MD, FRCPC, GRant D MaClean, MB, ChB, FRACP
}

BR YACYSHYN, GD MACLEAN. Tumour markers in gastrointestinal malignancy: What use to the clinician? Can J Gastroenterol 1992:6(6):329-333. A number of tumour markers have been identified in patients with gastrointestinal malignancies. Although these markers have limited clinical application, a knowledge of their use and shortcomings will help prepare for newer applications in gastrointestinal oncology. Tumour markers recently have been used as a serological means of monitoring gastrointestinal malignancy for recurrence and have been studied experimentally by radionucleotide imaging using radiolabelled markers. More recently, the use of tumour antigens as specific cancer vaccines has attracted the attention of gastrointestinal oncologists as therapeutic agents.

Key Words: Active specific immunotherapy, Gastrointestinal malignancy, Tumour markers

\section{De l'utilité des marqueurs de tumeurs pour le clinicien dans le cancer des voies digestives}

Un certain nombre de marqueurs de tumeurs ont été identifiés chez des gatients atteints de cancer des voies digestives. Bien que ces marqueurs aient une application clinique limitée, une connaissance de leur utilisation et de leurs inconvénients contribuera au développement de nouvelles applications en oncologie gastrointestinale. Les marqueurs des tumeurs ont récemment été utilisés à titre de méthode sérologique pour surveiller les récidives dans les cas de cancer des voies digestives et ont été étudiés expétimentalement par l'imagerie à l'aide de radio-nucléotides utilisant des marqueurs radio-actifs. Plus récemment, l'utilisation d'antigènes anti-tumoraux à titre de vaccins contre certains cancers spécifiques a attiré l'attention des oncologues spécialistes des voies digestives, sur leur emploi comme agents thérapeutiques.

Departments of Medicine, University of Alberta and Cross Cancer Institute, Edmonton, Alberta Correspondence and reprints: Dr B Yacyshyn, Department of Medicine, Division of Gastroenterology, 2E3.11 Walter Mackenzie Health Sciences Centre, University of Alberta, Edmonton, Alberta T6G 2R7. Telephone (403) 492-5957, Fax (403) $492-3340$

Received for publication May 3, 1992. Accepted June 9, 1992
$\mathrm{D}$ JRING THE PAST DECADE, ADvances in chemistry and hybridoma technology have enabled the identification of cancer-associated antigens. What do these tumour markers mean? Do they have clinical relevance? Can they be used for seromonitoring cancer patients? What has been the impact on the management of gastrointestinal malignancies? In addition to assays for carcinoembryonic antigen (CEA) and alpha fetoprotein, there are now assays for such markers as CA 19-9 and CA 125. However, claims for their utility have met with a mixture of enthusiasm and cynicism.

Most of the recently described tumour markers are glycoproteins or glycolipids. These are not cancer-specific. However, there are quantitative or qualitative changes in the expression of certain glycoconjugates; many of these changes arise early during malignant transformation. Hakomori (1), in reviewing the aberrant glycosylation found in cancer cells, has described these tumour markers as likely arising from abnormal activation of glycosyl transferases or incomplete glycosylation with precursor accumulation. As Hakomori suggests, this aberrant glycosyla- 
tion is probably the end result of altered genes or oncogenes. Some of the markers seen on cancer cells are normally cryptic within the membrane of normal cells and may also appear as a result of membrane conformational changes. However, some of the markers are simply expressed in increased quantities compared with normal counterpart cells, while others are expressed by cells where the normal counterpart cells do not express such antigens but where the same antigens can be found on other cells elsewhere in the body (for example, the expression of blood group antigens in colorectal cancer cells).

Old's central hypothesis shifted the focus in immuno-oncology from arguments around 'immune surveillance' to acceptance of the basic tenet - the existence of tumour-associated antigens (2). While earlier research had focused on transplantation antigens and molecules related to the major histocompatibility (MHC) antigens, interest in the past 20 years has focused on the abnormal expression of glycoconjugates and peptides which likely result from gene rearrangements or oncogenes (3).

Study of the expression of cancer-associated antigens has also created new opportunities for detection of metastases using monoclonal antibodies and immunoscintigraphy, and has enabled development of research into the potential of active specific immunotherapy using 'cancer vaccines'. How might this impact the management of gastrointestinal cancers?

\section{BACKGROUND}

Various antigens have been proposed and tested for clinical diagnosis and monitoring of disease states, including cancer. These include: normal differentiation antigens, MHC antigens, viral antigens, oncogene-related antigens, oncofetal antigens and other glycolipid or glycoprotein antigens. Normal differentiation antigens include $\mathrm{T}, \mathrm{B}$ and cellular activation markers, clinically used to diagnose and follow lymphoma. $\mathrm{MHC}$ antigens, clinically important in rejection of transplanted tissue, have been described as being expressed in colorectal carcinoma (4) as well as in the intestinal mucosa in inflammatory bowel disease and celiac sprue (5).

Viral antigens, in particular hepatitis B, are strongly associated with hepatocellular carcinoma, which also is associated with the oncofetal antigen Alpha-1-fetoprotein.

Oncogenes, or activated proto-oncogenes, may be markers of a premalignant state (6-8). The knowledge that cellular oncogenes play a role in carcinogenesis has led to their study as diagnostic tools, for example, in colorectal carcinoma where the RAS family of oncogenes has been studied. To date, they are more important from a pathological perspective in neoplasia and have not evolved a diagnostic role (9).

Altered carbohydrate molecules have been found on tumour cells $(1,10,11)$. Some of these markers are related to human blood group antigens including Lewis (Le) and the normally cryptic Thomsen-Freidenreich (TF) antigen, a precursor of the MN blood group antigens (42).

Recent reviews have described the appearance of TF and its related epitopes $\mathrm{Tn}$ and STn early during malig. nant transformation in colonic mucosa, suggesting their utility in the early histological detection of gastrointestinal tract cancers $(1,10)$. Of clinical relevance, TF expression on cancer cells has been associated with tumour aggressiveness and metastasis $(12,13)$, possibly in the same way adhesion molecules facilitate lymphocyte interactions (14).

\section{SERUM MARKERS IN CLINICAL USE}

Some of the cell-surface cancer-associated glycoconjugates appear to be useful targets for the detection of metastatic disease using monoclonal antibodies and immunoscintigraphy $(15-17)$. When shed into serum, some of these markers may have functional significance, including immunosuppressive effects (18). However, these serum tumour markers may also be useful for monitoring disease progression or response to therapy (19). Numerous serological tests with potential for monitoring or detecting malignancy have been described. Several of these are now in routine clinical use - eg, CA 125 in monitoring ovarian cancers, prostate specific acid phosphatase in monitoring prostate cancer, and $\beta$ human chorionic gonadotrophin and alpha fetoprotein in monitoring germ cell cancers.

With specific relevance to the management of gastrointestinal tract cancers, there are three areas where results to date have been encouraging: the immunohistochemical identification of cancers using monoclonal antibodies in vitro $(20)$; radioimmunodetection of metastases using radiolabelled monoclonal antibodies (21); and the potential for seromonitoring with monoclonal antibodies which detect elevated serum levels of newly identified cancer-associated antigens in patients with metastatic disease (22).

New markers, identified by monoclonal antibodies, currently being studied for utility in managing gastrointestinal tract cancers include CA 15 . 3, CA $19-9$ and CA 125, and may add to the markers currently used, CEA and alpha fetoprotein.

The key question has been whether these markers have sufficient specificity and sensitivity to be clinically useful. Because of the heterogeneity of individual cancers and their expression of these markers, recent reviews have described evaluating combinations of tumour markers in the hope of improving sensitivity, specificity and clinical utility. In breast cancer, it appears that combinations of markers may have greater specificity and predictive value $(23,24)$. For colorectal cancer, elevations of CEA $(43 \%)$ and CA $19-9$ $(34 \%)$ are not as predictive as when combined with CA 125 and SLEX (sialylated Lewis epitope). However, the predictive value was still only $57 \%$ in one study (22).

Ratios of various markers have been studied for their diagnostic or prognostic value. For example, in the diagnosis of carcinoma of unknown primary using logarithmic ratios, it has been proposed that the ratio of CA $15-3$ to CEA can be used to separate breast carcinoma from colorectal cancer (25). 


\section{SERUM TUMOUR MARKERS FOR GASTROINTESTINAL CANCERS}

Carcinoembryonic antigen: This wellknown family of related glycoproteins was first recognized by an antibody produced in rabbits vaccinated with human colonic adenocarcinoma (26). Since the development of sensitive radioassays, it has become apparent that elevations of CEA occur in a number of benign conditions including severe liver disease, inflammatory lesions (especially of the gastrointestinal tract), trauma, sepsis, infarction, renal impairment, collagen-vascular diseases and smoking (27). Its expression is heterogeneous by cancers and fluctuating trends have proven difficult to interpret in clinical monitoring, but it is the best studied marker in gastrointestinal oncolocgy. Due to its relatively low specificity and sensitivity, however, CEA is not useful as a sole diagnostic test for previously undetected cancer (28). Monitoring of CEA has been claimed to be of benefit in patients with elevated preoperative CEA levels (29). While it is a common practice to evaluate $\mathrm{CEA}$ levels regularly, for example every three months, there have been no studies which have confirmed that this is beneficial to patients. However, the lack of benefit may reflect the lack of currently useful systemic therapy for colorectal cancers, as well as the heterogenous expression of CEA (with consequent misleading trends).

CA 19-9: The antigen CA 19-9 is the sialylated form of the Lewis A antigen. It has been found in increased quantities in serum of patients with colorectal, gastric and pancreatic cancer (30-32). However, while patients with bulky cancers often have elevated CA 19-9 levels, most patients with early stage cancers have normal levels. Thus, there is limited value in trying to monitor patients for the early detection of relapse.

Among normal individuals, the highest values were found in women aged 10 to 40 years. Elevated values were also seen with pregnancy (33), suggesting a relationship with hormonal changes. Smokers' values are reportedly only minimally different from nonsmokers' (34).
Several benign diseases have CA 19-9 levels elevated above those found in normal individuals. Examples include cirrhosis $(35,36)$, cholestasis $(33,35,36)$, acute renal failure (35), peritonitis (36), diabetes (35) and various autoimmune (33) and endocrine diseases (36). Interestingly, $5 \%$ of the North American population is Lewis $\mathrm{a}-\mathrm{b}-$ and may be unable to produce CA 19-9 (37).

CA $19-9$ is elevated in 27 to $60 \%$ of patients with gastric cancer with a higher sensitivity (37\%) and specificity (80 to $100 \%$ ) than CEA $(38-40)$. In patients with carcinoina of the pancreas, CA 19-9 has a positive predictive value of $59 \%$ and a negative predictive value of $92 \%$ (41). As a single marker, it has not been proven to have clinical utility for seromonitoring. Perhaps it will be of use in a combined panel of diagnostic markers or as a target antigen or radioimmunodetection (42).

CA 125: This antigen, first described using the monoclonal antibody OC 125, appears to be a high molecular weight glycoprotein (43) which has been identified in colonic epithelium as well as pelvic organs and the peritoneal cavity. Elevated levels of CA 125 have been reported in $80 \%$ of patients with epithelial ovarian cancer (43) and emphasis has been on its use as a serum marker for monitoring patients with nonmucinous ovarian cancer $(19,44)$. CA 125 is present in cervical mucous and has been found elevated during menstruation and pregnancy (44-46). In addition, serum antigen elevations occur in patients with benign and malignant ascites $(48,49)$, and have been found elevated following heart failure and even infectious mononucleosis (unpublished data).

Elevated levels have also been found in serum of patients with carcinoma of the pancreas, lung, breast and gastrointestinal tract. However, CA 125 has been disappointing as a diagnostic marker for gastrointestinal malignancy. In studies to date, its greatest potential appears to be in combination with other markers such as CEA, CA 19-9 and SLEX in the management of colorectal carcinoma (22).
CA 15-3: CA 15-3 is another high molecular weight glycoprotein. Attempts have been made to use CA 15-3 to follow patients or to determine prognosis in patients with metastatic breast cancer. Elevated levels in patients with benign liver disease have been shown $(23,24)$. To date this marker's specificity has precluded it from being used routinely in nonselected clinical populations, and it has not been shown to have clinical utility as a single marker for seromonitoring patients with gastrointestinal cancers.

CA 50: CA 50, a more recently described marker for gastrointestinal tract cancers, appears to be related closely to CA 19-9 and has been described as the afucosyl form of the sialylated Lewis antigen. Its clinical use is still experimental.

\section{SCREENING}

Because of lack of necessary specificity and sensitivity, no tumour marker has yet been shown to be costeffective in population screening. Furthermore, none has been shown to be reliable in reaching a definitive diagnosis in a patient with metastatic adenocarcinoma of unknown primary. Therefore, caution should be taken in making assumptions when managing a patient with cancer metastatic to the gastrointestinal tract who is found, for example, to have an elevated CA 125 level. Similarly, a metastasis associated with elevated CEA is not necessarily gastrointestinal in origin. Perhaps the greatest impediment to using serum tumour markers in patients with gastrointestinal tract cancers effectively is the lack of chemotherapy effective against metastatic disease. Are there any therapeutic alternatives?

Active specific immunotherapy: The expression of altered carbohydrate molecules expressed on tumour cells has led to speculation that these might be useful immunogens and targets for active specific immunotherapy (ASI). Recent work has shown that shed tumour markers may be immunosuppressive, inhibiting an effective immune response against the cancer. An Edmonton based group is studying, in both animal 
models and early clinical trials, the potential of synthetic glycoconjugates for ASI in adenocarcinomas $(14,18)$. Cyclophosphamide is used prior to ASI in an attempt to inhibit the suppressor $\mathrm{T}$ cell activity induced by the shed serum mucin-associated glycoconjugates.

In the studies to date of 23 patients with metastatic ovarian or breast cancer, toxicity has been minimal. Most patients have developed haptenspecific immune responses following ASI, with in vitro studies showing cytotoxicity for relevant tumour cells. Some patients with early metastatic breast cancer (or minimal disease burden) appear to have had clinical or radiological responses to this ASI using synthetic 'vaccines'. The significance of these responses cannot be analyzed in these phase 1 studies, but phase II studies are planned for patients with low disease burden from metastatic breast or colorectal cancers. This approach adds a new dimension to the

ACKNOWLEDGEMENTS: Many thanks to Wendy McEachern for expert secretarial assistance in preparation of this manuscript.

\section{REFERENCES}

1. Hakomori S. Aberrant glycosylation in cancer cell membranes as focused on glycolipids: Overview and perspectives. Cancer Res 1983;45:2405.

2. Old L], Boyse EA. Immunology of experimental tumors. Ann Rev Med 1964:15:167.

3. Wortzel RD, Phillips C, Schreiber H. Multiple tumor specific antigens expressed on a single tumour cell. Nature 1983;304:165.

4. Daar AS, Fuggle SV, Ting A, Fabre JW. Anomalous expression of HDL-DR on human colorectal cancer cells. J Immunol 1982;129:447.

5. Scott H, Solheim BG, Brandtzaeg P, Thorsby E. HLA-DR like antigens in the epithelium of the human small intestine. Scand I Immunol 1980;12:77.

6. DuBois GC, Law LW, Appella E. Purification and biochemical properties of tumor-associated antigens from metliylcholanthrene induced murine sarcomas. Proc Natl Sci 1982;79:7669.

7. Cooper GM. Cellular transforming genes. Science 1982;217:801.

8. Land H, Parada LF, Weinberg RA. study of tumour markers in colorectal cancers. Are there markers relevant in suppressing most immunity to the cancer? Are these markers clues to effective ASI formulations? Seromonitoring will become relevant if ASI proves to have a clinical role in the therapy of gastrointestinal cancers.

\section{CONCLUSION}

In the past two decades, an awareness of aberrant glycosylation in cancers and the potential of hybridoma technology has led to a renewed optimism in the search for tumour markers associated with gastrointestinal cancers. The 'gold standard' has been CEA. While this antigen has not proven as useful clinically as originally hoped, it has served as a reminder and a challenge that gastrointestinal cancers may have quantitatively or qualitatively increased expression of glycoproteins and glycolipids which may have future clinical utility. Recently described monoclonal antibodies against

Cellular oncogenes and multistep carcinogenesis. Science 1983;222:771.

9. Trainer DL, Kline T, McCabe FL, et al. Biological characterization and oncogene expression in human colorectal carcinoma cell lines. Int J Cancer 1988;41:287.

10. Itzkowitz SH, Bloom EJ, Lokal WA, et al. A novel mucin antien associated with prognosis in colorectal cancer patients. Cancer 1990;9:160.

11. Hakomori S. Glycosphingolipids in cellular interaction, differentiation and oncogenesis. Ann Rev Biochem 1981;50:733.

12. Summers JL, Coon JS, Ward RM, Falor WH, Miller AW III, Weinstein RS. Prognosis in carcinoma of the urinary bladder based upon tissue blood group $\mathrm{ABH}$ and ThomsenFreidenreich antigen status and karyotype of the initial tumor. Cancer Res 1983;48:934-9.

13. Dow JA, di Sant'Agneses P, Cockett ATK. Expression of blood group precursor $T$-antigen as a prognostic marker for human bladder cancer treated by bacillus calmette-guerin and interleukin-2.J Urol 1989;142:978-82.

14. Springer TA. Adhesion receptors of the immune system. Nature 1990;346:425-34.

15. MacLean GD, Longenecker BM. Clinical significance of the ThomsenFreidenreich antigen. Semin Cancer markers such as CA 19-9, CA 125 and CA 50 have enabled study of the potential of these markers in seromonitoring, for identification of response to therapy and for early detection of relapse or recurrence of disease after treatment. These same markers may also prove useful as targets for immunodetection of metastatic disease using radiolabelled monoclonal antibodies and immunoscintography.

An exciting area of tumour marker application is the new field of ASI. Results from phase I studies in patients with ovarian carcinoma and breast cancer suggest the value of phase II studies to determine the response rate to this novel therapy. The study of tumour markers has previously focused on the potential of such markers for seromonitoring. Perhaps the detection of these new markers is providing the opportunity to understand better malignancy and to try much needed alternative management strategies for gastrointestinal cancers.

Biol 1991;2:433-9.

16. Samuel J, Noujaim AA, MacLean GD, Suresh MR, Longnecker BM. Analysis of human tumour associated Thomsen-Freidenreich antigen Cancer Res 1990;50:4801-8.

17. McEwan AJ, Wyeth P, Ackery D. Radioiodinated iodobenzyl guanidines for diagnosis and therapy. Int J Rad Appl Instrum 1986;37:765-75.

18. MacLean GD, Bowen-Yacyshyn MB, Samuel J, et al. Active immunization of human ovarian cancer patients against common carcinoina

(Thomsen-Freidenreich) determinant using a synthetic carbohydrate antigen. J Immunol. (In press)

19. Capstick V, MacLean GD, Suresh MR, et al. Clinical evaluation for a new two-site assay for CA125 antien. Int J Biol Markers 1991;6:129-35.

20. Yuan M, Itzkowitz SH, Ferrell LD, et al. Expression of Lewis $\mathrm{X}$ and sialylated Lewis $X$ antigens in human colorectal polyps. J Natl Cancer Inst 1987;78:479.

21. Holting T, Schlag P, Steinbacher M, Kretzchmar U, Georgi P, Herfarth C. The value of immunoscintography for the operative retreatment of colorectal cancer. Cancer 1989;64:830

22. Kawahard M, Terasaki PI, Chia D, Johnson C, Hermes M, Tokuta K Use of four monoclonal antibodies to detect tumor markers. Cancer $1986 ; 58: 2008$ 
23. Haynes DF, Zurawski VR, Kufe W. Comparison of circulating CA 15-3 and carcinoembryonic antigen levels in patients with breast cancer. J Clin Oncol 1986;4:1542.

24. Gion M, Mione R, Dittadi R, et al. Elevation of CA 15-3 levels in breast cancer patients. J Nucl Med All Sci 1986;30:29.

25. Wu JT. Expression of monoclonal antibody-defined tumor markers in four carcinomas. Ann Clin Lab Med 1989;19:17.

26. Gold P, Freedman SD. Specific carcinoembryonic antigens of human digestive system. J Exp Med 1965;122:468.

27. Lowenstein MS, Zamcheck N. Carcinoembryonic antigen (CEA) levels in benign gastrointestinal disease states. Cancer 1978;42:1412.

28. Fletcher RH. Carcinoembryonic antigen. In: Sox HC, ed. Common diagnostic tests: Use and interpretation. Philadelphia: American College of Physicians, 1987:257.

29. Arnaud JP, Koehl C, Adloff M. Carcinoembryonic antigen (CEA) in diagnosis and prognosis of colorectal carcinoma. Dis Colon Rectum 1980;23:141.

30. Magnani JL, Steplewski Z, Koprowski $\mathrm{H}$. Identification of the gastrointestinal and pancreatic cancer associated antigen detected by monoclonal antibody $19-9$ in the sera of patients as a mucin. Cancer Res 1983;43:5489

31. Koprowski H, Sears JF, Herlyn M. Sera from patients with adenocarcinoma of the colon inhibit binding of a monoclonal antibody to colon carcinoma cells. Science 1981;212:53.

32. Herlyn M, Sears HF, Steplewski Z.

Monoclonal antibody detection of a circulating tumour associated antien. I. Produce of antigen in sera of patients with colorectal, gastric and pancreatic carcinoma. J Clin Immunol 1982;2:135.

33. Del Villano BC, Zurawski VR. The carbohydrate 19-9 (CA 19-9): A monoclonal antibody defined tumor marker. In: Aloisi RM, ed. Immunodiagnostics. New York: Alan R Liss, 1983:269-282.

34. Green PJ, Ballas SK, Westskaemper P. CA 19-9 and CA 125 levels in the sera of normal blood donors in relation to smoking history. J Natl Cancer Inst $77: 337-41$

35. Arakawa Y, Ariga H, Kano M, et al. Determination and significance of a new carbohydrate antigen $19-9$ in digestive systemic cancers. Jap J Med 1985;24:121.

36. Encabo G, Ruibal A. Seric CA 19-9 levels in patients with non-tumoral pathologies: Our experience in 892 cases. Cancer 1991;73:256.

37. Haglund C, Roberts PJ, Kuusela P, et al. Evaluation of CA 19-9 as a serum tumor marker in pancreatic cancer. Br J Cancer 1986;53:197.

38. Bogenschutz $\mathrm{O}$, Brunmmendorf $\mathrm{T}$, Staab HJ, et al. Prognostic value of preoperative CEA levels compared to clinical staging IV. Histological grading and tumor type in colorectal and gastric cancer. J Surg Oncol $1986 ; 32: 165$.

39. Jalanko H, Haglund C, Roberts PJ, Kuusela P. Tumor markers in gastrointestinal cancers. In: Holmgren
J, ed. Tumour Marker Antigens.

Sweden: Nordbok, 1985:114.

40. Staab JH, Anderer FA, Brummendorf $\mathrm{T}$, et al. Prognostic value of preoperative serum CEA level compared to clinical staging II stomach cancer. Br J Cancer 1982;45:718.

41. Pleskow DK, Berger HJ, Gyves J, et al. Evaluation of a serologic marker CA $19-9$ in the diagnosis of pancreatic cancer. Ann Intern Med 1989:110:704.

42. Silverstein MD, Richter JM, Podolsky DK, Warshaw AL. Suspected pancreatic cancer presenting as pain or weight loss. Analysis of diagnostic strategies. World J Surg 1984:8:839.

43. Bast RC, Klug TL, St John E, et al. A radioimmunooassay using a monoclonal antibody to monitor the course of epithelial ovarian cancer. N Engl J Med 1983;309:883.

44. Bast RC, Feenney M, Iazarus $H$, et al. Reactivity of a monoclonal antibody with human ovarian carcinoma. J Clin Invest 1981;68:1331.

45. Niloff JM, Klug TL, Schaetzl E, et al. CA 125 antigen levels in obstetric and gynecologic patients. Ohstet Gynecol 1984;64:703.

46. Haga Y, Sakamoto K, Egami H, et al. Evaluation of serum CA 125 values in healthy individuals and pregnant women. Am J Med Sci 1986:29:30-4.

47. Bergmann JF, Bidart JM, George M, et al. Elevation of CA 125 in patients with benign and malignant ascites. Cancer 1987;59:213.

48. Mezger J, Willams W, Lamerz R. Elevated serum CA 125 levels in patients with benign ascitic or pleural effusions. Tumour Biol 1988;9:47. 


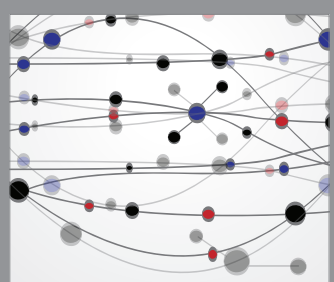

The Scientific World Journal
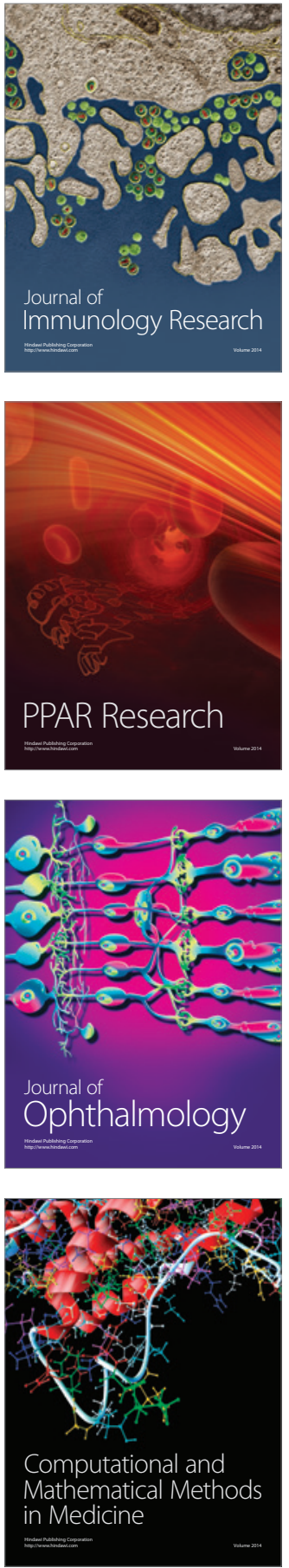

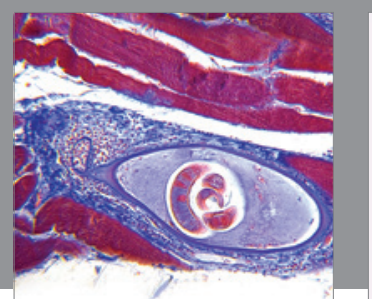

Gastroenterology Research and Practice

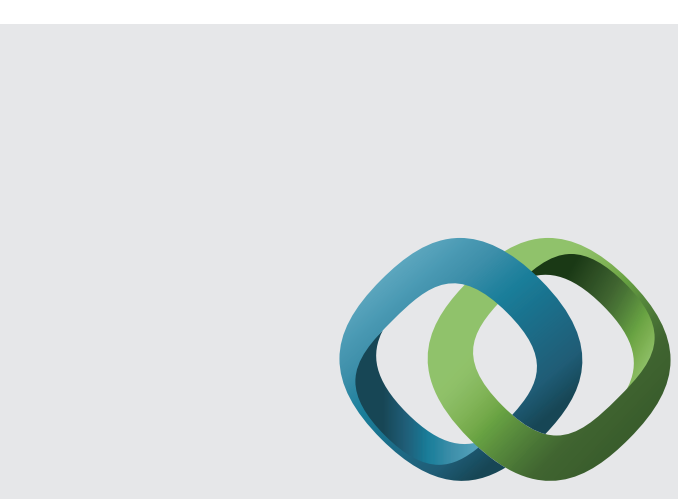

\section{Hindawi}

Submit your manuscripts at

http://www.hindawi.com
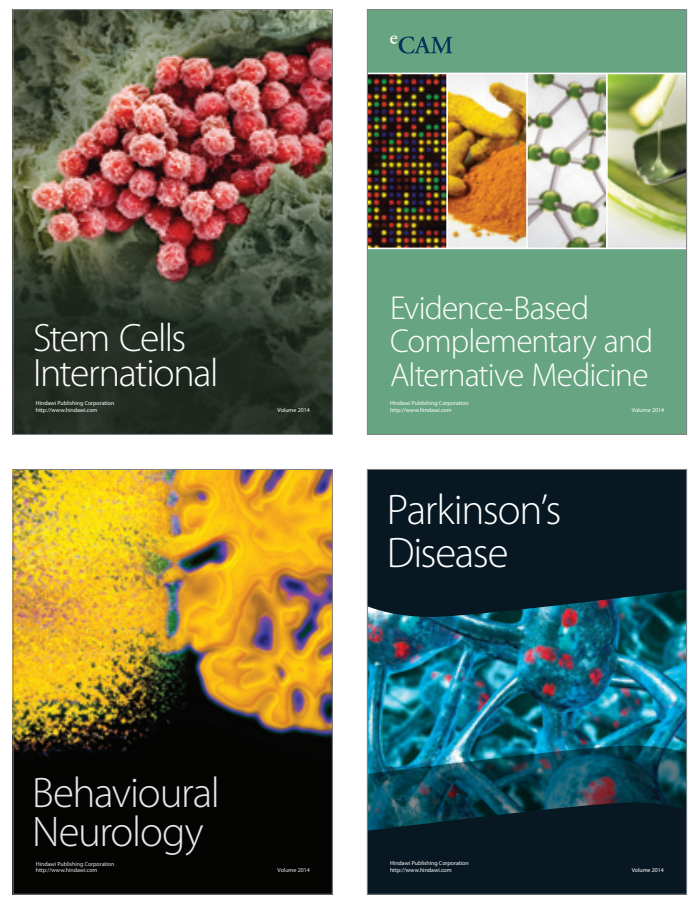
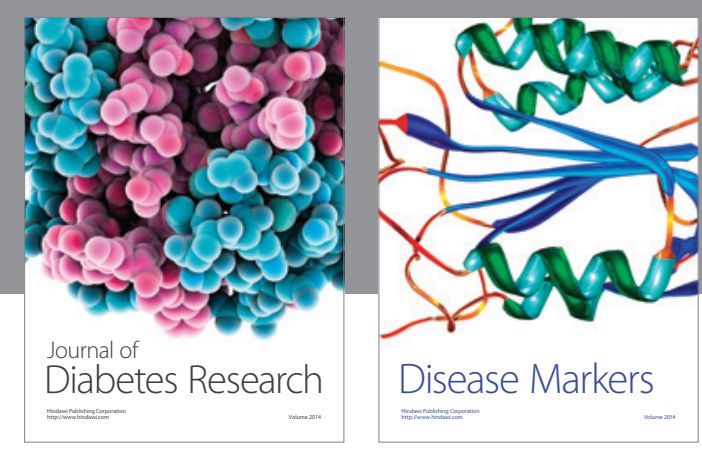

Disease Markers
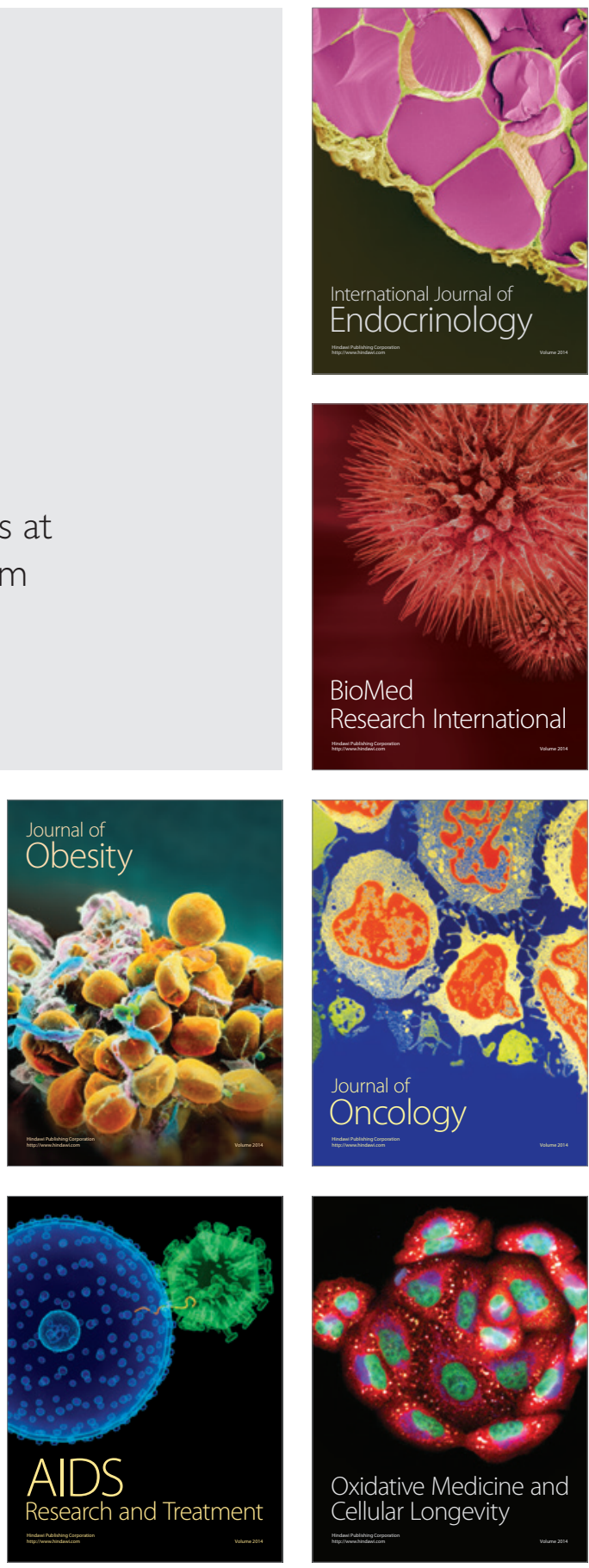